\title{
Ammonia Gas Detection under Various Humidity Conditions Using Waveguide Surface Plasmon Resonance Spectroscopy
}

\author{
Kazunari Shinbo, ${ }^{\dagger}$ Ryo Komai, Hiroki Honda, Yasuo OhdaIra, Akira Baba, Keizo Kato, and \\ Futao KANEKo
}

Graduate School of Science and Technology and Center for Transdisciplinary Research, Niigata University, 2-8050 Ikarashi, Nishi, Niigata 950-2181, Japan

\begin{abstract}
Analysis of $\mathrm{NH}_{3}$ gas under various humidity conditions was conducted using a waveguide surface plasmon resonance (SPR) sensor with dual sensing parts. Two pairs of Ag films/sensing polymer films were prepared separately on a waveguide core of BK-7 slide glass. Poly(acrylic acid) (PAA) and poly(vinyl alcohol) (PVA) were used as sensing materials. A white light was guided through the core by illuminating the substrate edge, and the SPR property was investigated by observing the output light spectrum. The thicknesses of PAA and PVA films were adjusted to induce SPR at different wavelengths. PAA exhibited remarkable response against $\mathrm{NH}_{3}$ gas, but it also exhibited a strong dependence on humidity. In contrast, PVA responded to humidity but hardly responded to $\mathrm{NH}_{3}$ gas below $20 \mathrm{ppm}$. The dual sensing would allow us to conduct precise $\mathrm{NH}_{3}$ measurements under various humidity conditions.
\end{abstract}

Keywords Surface plasmon, gas sensor, waveguide, ammonia, poly(vinyl alcohol), poly(acrylic acid)

(Received January 6, 2017; Accepted March 1, 2017; Published April 10, 2017)

\section{Introduction}

Surface plasmon (SP) is a surface electromagnetic wave and is commonly observed on a noble metal surface. ${ }^{1-3}$ SP has many useful properties; for example, $\mathrm{SP}$ is accompanied by an intense electric field. The electric field can be used to enhance molecular excitation for photoluminescence and optical absorption in photovoltaic cells. ${ }^{4-7}$ The field exists in the vicinity of a metal surface, and the feature can be used to develop a nano-sized optical circuit. ${ }^{8}$ Conversion between SP and far-field light can be conducted by a prism or a periodic structure, ${ }^{9,10}$ and the phenomenon is promising for the development of novel optical devices and the improvement of organic light-emitting diode efficiency. ${ }^{11-13}$ Furthermore, SP excitation strongly depends on the dielectric constant and the thickness of metal film and adjacent media. Therefore, a surface plasmon resonance (SPR) technique is frequently employed in the development of sensitive sensors of bio- and gas-molecular adsorptions. ${ }^{14-19}$

SP is a coupling mode of a light and corrective oscillation of surface free electrons, and the SP excitation condition must be fulfilled for observation. SP excitation is commonly conducted by a Kretschmann configuration, ${ }^{1}$ like a glass prism, metal film, and dielectric film structure. A p-polarized light is totally reflected and an evanescent wave is produced in the setup. The wavenumber along the surface can be adjusted for SP excitation by the incident angle, and an attenuation of reflected light can be observed when SP excitation occurs. The use of a prism,

† To whom correspondence should be addressed.

E-mail: kshinbo@eng.niigata-u.ac.jp however, sometimes causes difficulties in preparing a compact sensor. It is also known that an optical waveguide with a metal film can be used for SP excitation..$^{20-23}$ The waveguide core instead of the prism is used, and the evanescent wave of the guided light induces SP excitation at the metal film surface. A compact sensor can be prepared since no prism coupler is needed, and the waveguide SPR sensor is quite simple and reasonable.

We reported a waveguide SPR gas sensor with multiple sensing parts. ${ }^{24,25}$ A white light was used in the setup, and the output light spectra exhibited dips (attenuations) at SPR wavelengths. The sensor allowed multiple observations without using a waveguide array, many light detectors or a more complicated setup. The experimental error between the sensing parts due to condition differences would be small since the sensors were on the same substrate. Additionally, the sensor can be used in vacuum, air or liquid, so it may be a powerful tool for evaluating adsorption phenomena.

$\mathrm{NH}_{3}$ is widely used, for example, to produce fertilizers and synthesize various chemical compounds; however, it is harmful, combustible and is a common air pollutant. Therefore, a sensitive $\mathrm{NH}_{3}$ gas detection method is in high demand for safety and environmental monitoring. Furthermore, a sensitive $\mathrm{NH}_{3}$ gas sensor is needed in medical diagnostics. ${ }^{26}$ We reported a fundamental study of the $\mathrm{NH}_{3}$ gas responses of sensors with dual sensing parts consisting of poly(acrylic acid) (PAA) and poly(vinyl alcohol) (PVA) sensing materials. ${ }^{25}$ PAA responded well to $\mathrm{NH}_{3}$ gas, however also exhibited strong humidity dependence. ${ }^{27}$ Practical $\mathrm{NH}_{3}$ gas sensors would be used in atmospheres with various humidity conditions; therefore, simultaneous humidity measurement was necessary for accurate $\mathrm{NH}_{3}$ gas detection. We found PVA was useful for humidity 


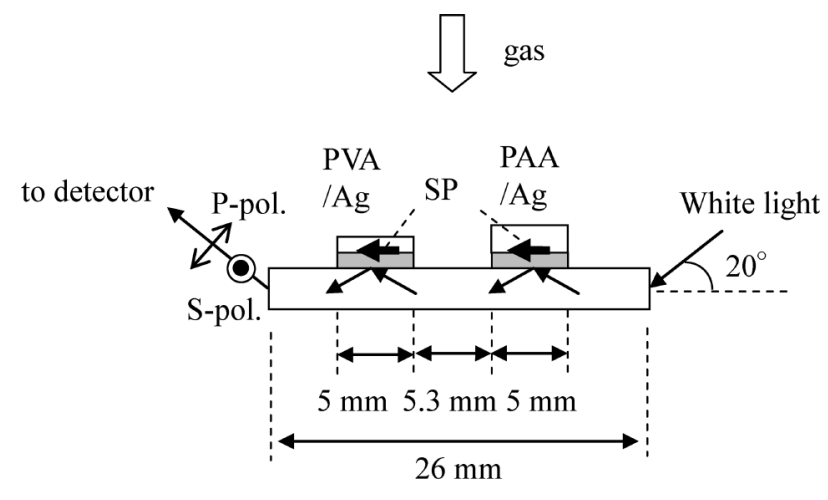

Fig. 1 Sensor structure.

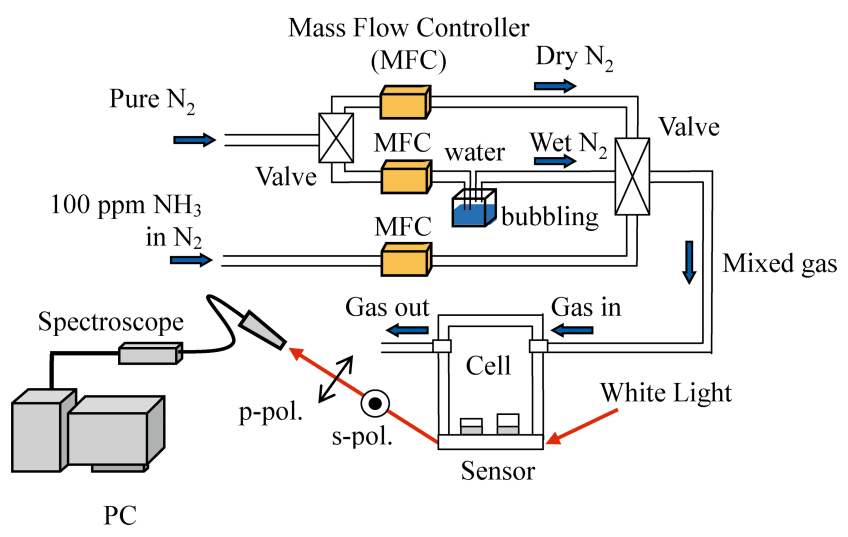

Fig. 2 Experimental setup.

measurement in the previous study. ${ }^{25}$ In this paper, investigations were primarily conducted to clarify responses to $\mathrm{NH}_{3}$ gas with low concentration $(5-20 \mathrm{ppm})$ under various humidity conditions.

\section{Experimental}

Figure 1 shows the sensor structure. A 1-mm thick BK-7 glass substrate was used as a slab waveguide, and $50 \mathrm{~nm}$ thick $\mathrm{Ag}$ thin films were vacuum-evaporated separately. Dual sensing parts with PVA film (PVA/Ag) and with PAA film (PAA/Ag) were prepared on the waveguide. PAA and PVA were purchased from Aldrich and were used as sensing materials for $\mathrm{NH}_{3}$ and humidity, respectively. The PAA and PVA films were spincoated only on the desired Ag film by masking the other part. The structure of the BK-7 substrate, Ag film, and dielectric film formed a Kretschmann configuration and worked as an SPR sensor. The substrate edge was illuminated by a white light at an incident angle of $20^{\circ}$. The output of s-polarized light at initial state in $\mathrm{N}_{2}$ was used as a reference $\left(I_{S}\right)$. The output of p-polarized light, which induced SP excitation, was also observed $\left(I_{\mathrm{P}}\right)$. The ratio of $I_{\mathrm{P}}$ and $I_{\mathrm{S}}\left(\right.$ i.e. $\left.\left(I_{\mathrm{P}} / I_{\mathrm{S}}\right) \times 100 \%\right)$, was calculated as an SPR curve. A dip was observed in the curve when the SP excitation occurred. The SPR wavelength qualitatively redshifted with increasing polymer thickness. The thicknesses of PAA and PVA films were adjusted to induce SPR at different wavelengths.

The experimental setup is shown in Fig. 2. Dry $\mathrm{N}_{2}$ gas, $\mathrm{N}_{2}$ gas bubbled through water (wet $\mathrm{N}_{2}$ gas), and $100 \mathrm{ppm} \mathrm{NH}_{3}$ gas in

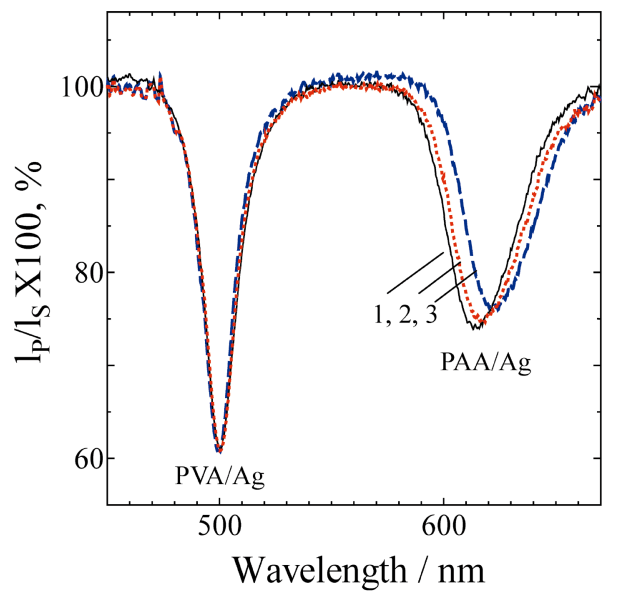

(a)

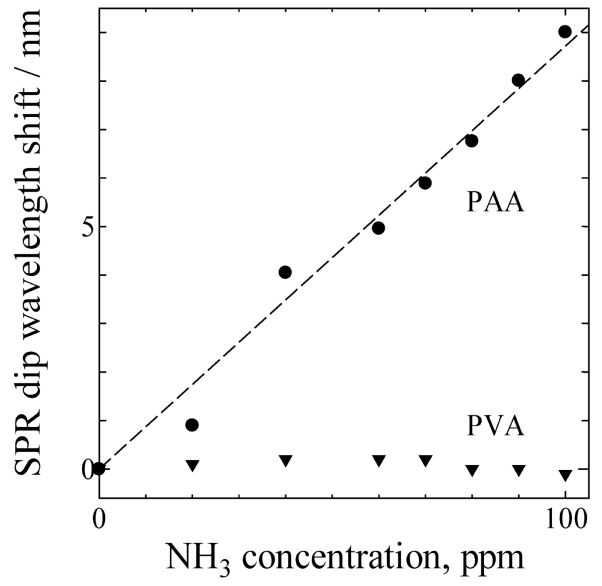

(b)

Fig. $3 \mathrm{NH}_{3}$ gas sorption and SPR property changes (under $0 \% \mathrm{RH}$ ): (a) SPR curves under 0 (curve 1), 40 (curve 2), and 100 (curve 3) ppm $\mathrm{NH}_{3}$; (b) SPR dip wavelength shift $(47.5 \mathrm{~nm}$ thick PVA and $84.7 \mathrm{~nm}$ thick PAA films).

$\mathrm{N}_{2}$ were mixed to control the concentration of $\mathrm{NH}_{3}$ and humidity. The flow rate of the mixed gas was $200 \mathrm{ml} / \mathrm{min}$. SPR measurements were performed at room temperature under atmospheric pressure. The spectrum of the output light was monitored using an HR4000HC spectrometer (Ocean Optics). WinSpall 2.20 software (Max-Plank-Institute for Polymer Research, Mainz, Germany) was used to obtain the SPR curves and evaluate the film thickness from the experimental SPR dip wavelengths. Refractive indices from the literature ${ }^{28-30}$ were used for the calculation.

\section{Results and Discussion}

The SPR curves under constant humidity $(0 \% \mathrm{RH})$ and various $\mathrm{NH}_{3}$ concentrations were observed as shown in Fig. 3(a). Curve 1 was obtained under $0 \mathrm{ppm} \mathrm{NH}_{3}$, and two dips at around 500 and $620 \mathrm{~nm}$ were observed. The film thicknesses of PVA and PAA films were estimated to be 47.5 and $84.7 \mathrm{~nm}$ from the dip wavelengths, respectively. As shown here, SPR dips can be separately observed by adjusting deposited film thickness. Curves 2 and 3 were observed under 40 and $100 \mathrm{ppm} \mathrm{NH}_{3}$, and the PAA/Ag dip redshifted against $\mathrm{NH}_{3}$ gas. PAA seemed to be promising for $\mathrm{NH}_{3}$ sensor, and $\mathrm{NH}_{3}$ molecules should react with 


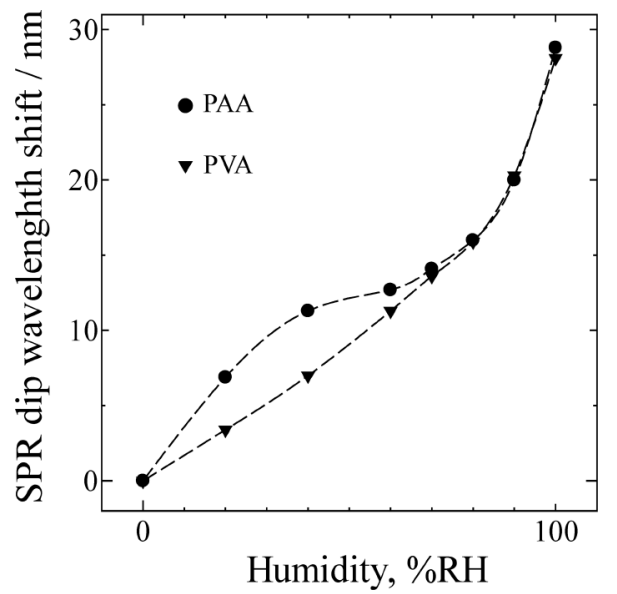

Fig. 4 SPR dip shift $v s$. humidity under $0 \mathrm{ppm} \mathrm{NH}_{3}(47.5 \mathrm{~nm}$ thick PVA film and $84.7 \mathrm{~nm}$ thick PAA film).

Table 1 SPR dip wavelength under various $\mathrm{NH}_{3}$ concentrations and humidity conditions $(56.7 \mathrm{~nm}$ thick PVA and $97.5 \mathrm{~nm}$ thick PAA)

\begin{tabular}{|c|c|c|}
\hline & \multicolumn{2}{|c|}{ SPR dip wavelength/nm } \\
\hline & PVA/Ag & $\mathrm{PAA} / \mathrm{Ag}$ \\
\hline $0 \% \mathrm{RH} \mathrm{N}_{2}$ & 515.4 & 655.1 \\
\hline $0 \% \mathrm{RH} \mathrm{N} \mathrm{N}_{2}$ with $50 \mathrm{ppm} \mathrm{\textrm {NH } _ { 3 }}$ & 516.1 & 671.3 \\
\hline $50 \% \mathrm{RH} \mathrm{N}_{2}$ & 519.9 & 685.7 \\
\hline $50 \%$ RH N $N_{2}$ with $50 \mathrm{ppm} \mathrm{NH}_{3}$ & 522.2 & 756.0 \\
\hline
\end{tabular}

carboxylic groups of PAA. ${ }^{27}$ In contrast, PVA does not have carboxylic groups, and the PVA/Ag dip was found to exhibit almost no response to $\mathrm{NH}_{3}$ gas. The SPR dip shifts of PAA/Ag and PVA/Ag are shown in Fig. 3(b). The shift was set as positive for a redshift. The PAA/Ag dip redshifted with an increase of $\mathrm{NH}_{3}$ concentration, and the response was almost linear in this condition. The linear fitted line (broken line) is also shown in the figure, as is the R-squared value of 0.9839 . In contrast, the PVA/Ag dip response was barely in the concentration range.

Figure 4 shows the SPR dip wavelength shift of the same sample under various humidity conditions without $\mathrm{NH}_{3}$. Clearly, the dips redshifted with humidity. Both PAA and PVA are water soluble, so water vapor sorption in the films easily occurred and induced redshifts of the dip wavelength. A linear relationship was not observed here.

Fundamental responses to $\mathrm{NH}_{3}$ under various humidity conditions were studied for the sensor with $56.7 \mathrm{~nm}$ thick PVA and $97.5 \mathrm{~nm}$ thick PAA films, and the results are shown in Table 1.25 The initial SPR dips under $0 \% \mathrm{RH} \mathrm{N} \mathrm{N}_{2}$ without $\mathrm{NH}_{3}$ were observed at 515.4 and $655.1 \mathrm{~nm}$. The shifts of dip wavelength due to introductions of $0 \% \mathrm{RH} \mathrm{N} \mathrm{N}_{2}$ with $50 \mathrm{ppm} \mathrm{NH}_{3}$,

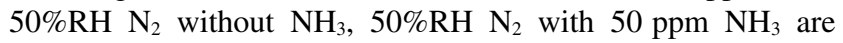
shown. The PAA/Ag dip redshifted against $\mathrm{NH}_{3}$ gas, and a remarkable response was seen for introduction to $50 \% \mathrm{RH} \mathrm{N}_{2}$ with $50 \mathrm{ppm} \mathrm{NH}_{3}$. It has been reported that the reaction between $\mathrm{NH}_{3}$ and the carboxyl groups of PAA destroys the PAA interchain hydrogen bonds and enhances water sorption. ${ }^{31}$ The enhanced water sorption would induce the remarkable PAA/Ag dip shift shown in Table 1. In contrast, the PVA/Ag dip did not show a remarkable difference, regardless of $\mathrm{NH}_{3}$ existence.

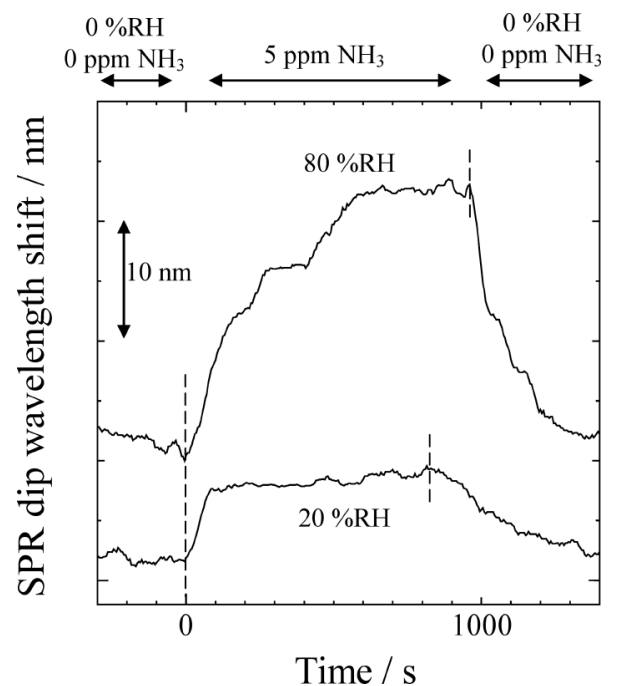

Fig. 5 Kinetic responses of SPR dip wavelength shift for 20 and $80 \% \mathrm{RH} \mathrm{N}_{2}$ with $20 \mathrm{ppm} \mathrm{NH}_{3}$ (84.7 nm thick PAA film).

Table 2 Response times for various gas introductions $(56.7 \mathrm{~nm}$ thick PVA and $97.5 \mathrm{~nm}$ thick PAA)

\begin{tabular}{lcccr}
\hline & \multicolumn{4}{c}{ Response time/s } \\
\cline { 2 - 5 } & Humidity, \%RH & \multicolumn{2}{c}{$\mathrm{NH}_{3}$ concentration, ppm } \\
\cline { 3 - 5 } & & 5 & 10 & 20 \\
\hline PAA/Ag & 20 & 189 & 302 & 214 \\
& 40 & 304 & 305 & 558 \\
PVA/Ag & 80 & 503 & 1280 & 1310 \\
& 20 & 368 & 275 & 360 \\
& 40 & 263 & 280 & 242 \\
& 80 & 411 & 420 & 497 \\
\hline
\end{tabular}

The PVA/Ag dip wavelength exhibited a small redshift against $\mathrm{NH}_{3}$ under $50 \% \mathrm{RH}$ due to dissolution of $\mathrm{NH}_{3}$ in water in the PVA film. PAA is promising as an $\mathrm{NH}_{3}$ sensing material, but humidity monitoring is also necessary, since the PAA/Ag response includes water vapor sorption. The PVA/Ag dip exhibited a weak response against $\mathrm{NH}_{3}$; therefore PVA would be useful for humidity monitoring in a $\mathrm{N}_{2}$ with $\mathrm{NH}_{3}$ gas.

The responses against various conditions for the sensor with $47.5 \mathrm{~nm}$ thick PVA and $84.7 \mathrm{~nm}$ thick PAA films were investigated. The kinetic responses of PAA/Ag dip against introductions of 20 and $80 \% \mathrm{RH} \mathrm{N} \mathrm{N}_{2}$ with $5 \mathrm{ppm} \mathrm{NH}_{3}$ gases are shown in Fig. 5. The dip shift saturated gradually, and the times to reach the value of $90 \%$ shift (response times) were 189 and $503 \mathrm{~s}$ for 20 and $80 \% \mathrm{RH} \mathrm{N} \mathrm{N}_{2}$ with $5 \mathrm{ppm} \mathrm{NH}_{3}$ gases, respectively. The responses for other conditions were also investigated, and the response times are summarized in Table 2. The response time tends to increase with humidity and $\mathrm{NH}_{3}$ concentration ( 214 and 1310 s for 20 and $80 \% \mathrm{RH} \mathrm{N} \mathrm{N}_{2}$ with $20 \mathrm{ppm} \mathrm{NH}_{3}$ gases, respectively). Furthermore, the redshift of peak wavelength exhibited a similar tendency, and the result seemed to be due to the amount of sorbed water and $\mathrm{NH}_{3}$ molecules. The response time was below $500 \mathrm{~s}$ for the PVA/Ag dip.

The responses against various conditions for the sensor are shown in Fig. 6. The $\mathrm{NH}_{3}$ concentration was adjusted in the range of $0-20 \mathrm{ppm}$. The PAA/Ag dip hardly responded against $\mathrm{NH}_{3}$ under $0 \% \mathrm{RH}$, but the dip shift increased with humidity. 


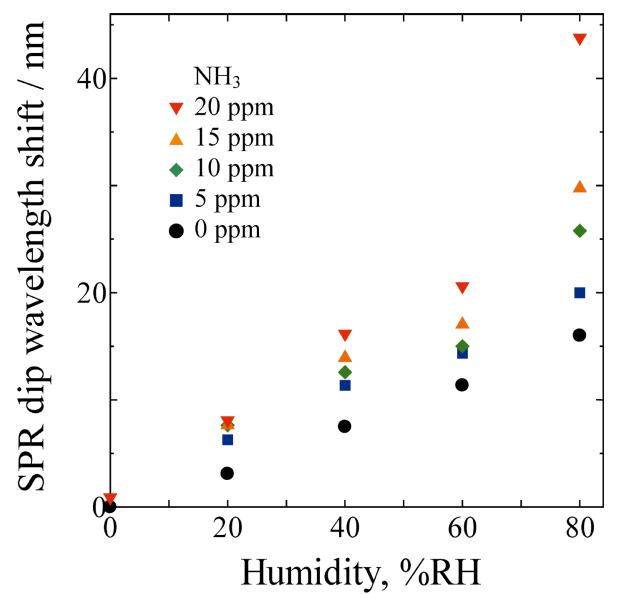

(a)

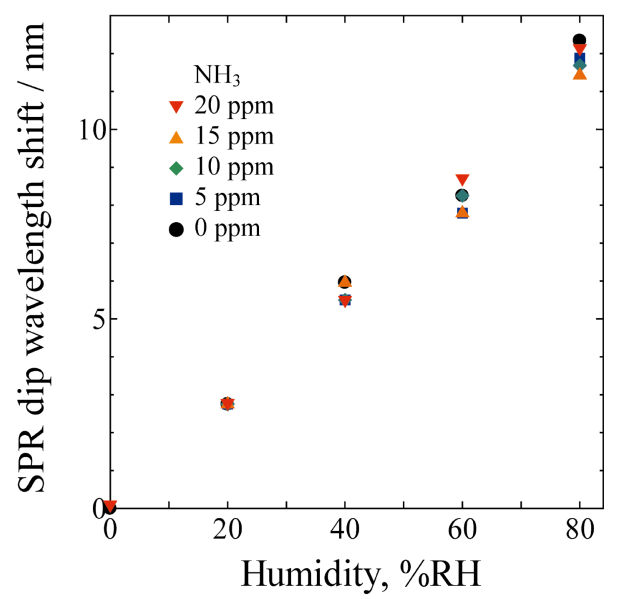

(b)

Fig. 6 SPR wavelength shift for various $\mathrm{NH}_{3}$ concentrations and humidity conditions: (a) $84.7 \mathrm{~nm}$ thick PAA and (b) $47.5 \mathrm{~nm}$ thick PVA.

Many water and $\mathrm{NH}_{3}$ molecules seemed to be sorbed under high humidity conditions, since the PAA hydrogen bonds were destroyed. The improved PAA sensitivity enabled $\mathrm{NH}_{3}$ detection around $1 \mathrm{ppm}$ under humidity of $40 \% \mathrm{RH}$ or more. The relationships for PVA dip were plotted in Fig. 6(b). The PVA/Ag dip did not exhibit a clear tendency against $\mathrm{NH}_{3}$; however, it almost responded to humidity linearly. Humidity in this range can be monitored through PVA results. The results are plotted again as dip wavelength shift vs. $\mathrm{NH}_{3}$ concentration relationships (for 20 and $80 \% \mathrm{RH}$ ) in Fig. 7. The PAA/Ag dip response exhibited a clear dependence on $\mathrm{NH}_{3}$ concentration and increased with humidity. The PVA/Ag dip was dependent on humidity and was almost independent of $\mathrm{NH}_{3}$ concentration in this range. The relationship between the PAA and PVA results allows the identification of humidity and $\mathrm{NH}_{3}$ concentration. By preparing a database of responses against $\mathrm{NH}_{3}$ and humidity, $\mathrm{NH}_{3}$ concentration can be estimated from the SPR results of PAA and PVA. The reproducibility and reliability of the sensor are now under investigation.

\section{Conclusions}

Simultaneous measurement of $\mathrm{NH}_{3}$ gas and humidity was

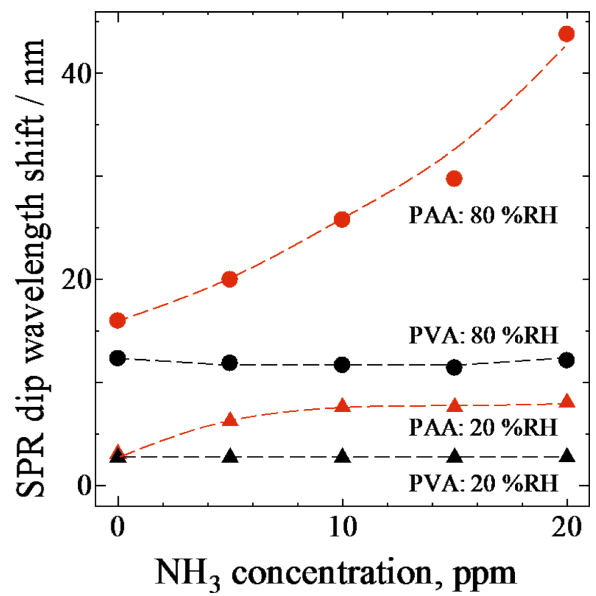

Fig. 7 SPR wavelength $v s$. $\mathrm{NH}_{3}$ concentration relations under 20 and $80 \%$ RH.

conducted using a waveguide SPR sensor. The dual sensing parts of PAA/Ag and PVA/Ag films were prepared on a single glass substrate, and dual SPR dip observations were possible in the structure. The PAA dip responded remarkably to $\mathrm{NH}_{3}$ gas containing water vapor, and it was attributed to the destruction of the hydrogen bond of PAA in the film by $\mathrm{NH}_{3}$ and enhancement of water sorption. In contrast, PVA markedly responded to water vapor only and exhibited a poor response against $\mathrm{NH}_{3}$; PVA was promising for monitoring humidity. The combination measurement of PVA and PAA allows the identification of $\mathrm{NH}_{3}$ concentration under various humidity conditions.

\section{Acknowledgements}

This work was partially supported by a Grant-in-Aid for Scientific Research (KAKENHI) from the Japan Society for the Promotion of Science (JSPS).

\section{References}

1. V. M. Agranovich and D. L. Mills (ed.), "Surface Polaritons", 1982, North-Holland, Amsterdam.

2. S. Kawata (ed.), "Near-Field Optics and Surface Plasmon Polaritons", 2001, Springer, Berlin.

3. V. M. Shalaev and S. Kawata (ed.), "Nanophotonics with Surface Plasmons", 2007, Elsevier, Amsterdam.

4. T. Liebermann and W. Knoll, Colloids Surf., A, 2000, 171, 115 .

5. T. Kume, S. Hayashi, and K. Yamamoto, Jpn. J. Appl. Phys., 1993, 32, 3486.

6. K. Shinbo, T. Ebe, F. Kaneko, K. Kato, and T. Wakamatsu, IEICE TRANS. ELECTRON., 2000, E83-C, 1081.

7. A. Baba, K. Wakatsuki, K. Shinbo, K. Kato, and F. Kaneko, J. Mater. Chem., 2011, 21, 16436.

8. A. Vakil and N. Engheta, Science, 2011, 332, 1291.

9. I. Pockrand, A. Brillante, and D. Möbius, Chem. Phys. Lett., 1980, 69, 499.

10. T. W. Ebbesen, H. J. Lezec, H. F. Ghaemi, T. Thio, and P. A. Wolff, Nature, 1998, 391, 667.

11. K. Shinbo, S. Toyoshima, Y. Ohdaira, K. Kato, and F. Kaneko, Jpn. J. Appl. Phys., 2005, 44, 599. 
12. J. Feng, T. Okamoto, and S. Kawata, Opt. Lett., 2005, 30, 2302.

13. J. Feng, T. Okamoto, R. Naraoka, and S. Kawata, Appl. Phys. Lett., 2008, 93, 051106.

14. J. Homola (ed.), "Surface Plasmon Resonance Based Sensors", 2006, Springer, Berlin.

15. M. Mitsushio and M. Higo, Anal. Sci., 2011, 27, 247.

16. T. Kawawaki, N. Shinjo, and T. Tatsuma, Anal. Sci., 2016, 32(3), 271.

17. K. Shinbo, H. Ishikawa, A. Baba, Y. Ohdaira, K. Kato, and F. Kaneko, Appl. Phys. Express, 2012, 5, 036603.

18. K. Shinbo, Y. Ohdaira, A. Baba, K. Kato, and F. Kaneko, Mol. Cryst. Liq. Cryst., 2015, 622, 67.

19. K. Shinbo, K. Takizawa, N. Obata, C. Lertvachirapaiboon, A. Baba, K. Kato, and F. Kaneko, Polym. Bull., 2016, 73, 2539.

20. S. Sano, H. Mastubara, M. Hashizume, Y. Matsui, and S. Shiokawa, IEEJ Trans. Sens. Micromach. Jpn., 1997, 117, 627.

21. R. Micheletto, K. Hamamoto, S. Kawai, and Y. Kawakami, Sens. Actuators, A, 2005, 119, 283.

22. J. Dostálek, J. Tyroký, J. Homola, E. Brynda, M. Skalský,
P. Nekvindová, J. Pirková, J. Kvor, and J. Schröfel, Sens. Actuators, B, 2001, 76, 8.

23. T. Matsushita, T. Nishikawa, H. Yamashita, J. Kishimoto, and Y. Okuno, Sens. Actuators, B, 2008, 129, 881.

24. K Shinbo, K. Mizusawa, H. Takahashi, Y. Ohdaira, A. Baba, K. Kato, F. Kaneko, and N. Miyadera, Jpn. J. Appl. Phys., 2011, 50, 01BC15.

25. R. Komai, H. Honda, A. Baba, K. Shinbo, K. Kato, and F. Kaneko, 7th International Symposium on Electrical Insulating Materials, 2014, VA27, 284.

26. B. Timmer, W. Olthuis, and A. Berg, Sens. Actuators, B, 2005, 107, 666.

27. B. Ding, M. Yamazakia, and S. Shiratori, Sens. Actuators, $B, \mathbf{2 0 0 5}, 106,477$.

28. F. Barroso-Bujans, R. Serna, E. Sow, J.L.G. Fierro, and M. Veith, Langmuir, 2009, 25(16), 9094.

29. D. Mergel, D. Buschendorf, S. Eggert, R. Grammes, and B. Samset, Thin Solid Films, 2000, 371, 218.

30. A. D. Rakić, A. B. Djurišic, J. M. Elazar, and M. L. Majewski, Appl. Opt., 1998, 37, 5271.

31. M. Hoerter, A. Oprea, N. Barsan, and U. Weimar, Sens. Actuators, B, 2008, 134, 743. 